\title{
Costo-EFECTIVIDAd de InSTRUmentos ECONÓMicos PARA EL CONTROL DE LA CONTAMINACIÓN. El CASO del Uso de LEÑA*
}

\author{
Carlos Chávez \\ Universidad de Concepción \\ WALTER GómeZ \\ Universidad de la Frontera \\ SANDRA BRICEÑO \\ Universidad de Concepción
}

We explore the cost-effectiveness of economic incentives to induce changes in wood consumption in urban areas. We consider the case of Temuco and Padre Las Casas in southern Chile. Three incentives schemes are considered: subsidy to the demand of dry wood, subsidy to the supply of dry wood, and enforcement of the standard on the humidity content of wood. The results suggest that subsidies should be targeted to the production of dry wood. The impacts on the aggregate level of emissions are small. Finally, enforcing the humidity standard is more cost-effective than the evaluated subsidy programs.

\section{JEL: Q28, Q21, H31}

Keywords: Política Ambiental, Costo-Efectividad, Incentivos Económicos, Contaminación Urbana.

\section{INTRODUCCIÓN}

Entre los diversos problemas ambientales que enfrenta Chile, se destaca el de la contaminación del aire en zonas urbanas, especialmente en regiones del centro y sur del país. No obstante que el problema de contaminación del aire de la ciudad de Santiago es el más conocido y está ampliamente documentado

* Chávez y Gómez agradecen el apoyo financiero proporcionado por CONICYT Chile, a través del Proyecto Fondecyt $\mathrm{N}^{\mathrm{o}}$ 1080287. Los autores agradecen, además, la valiosa información y fructíferas discusiones con el grupo del Área de Control de la Contaminación, CONAMA Región de La Araucanía.

Email: cchavez@udec.cl 
(ver por ejemplo CONAMA-DICTUC, 2007), los problemas asociados al deterioro de la calidad del aire se han extendido a regiones, aunque debido a causas muy diferentes (Celis et al., 2006 y 2004).

Este es el caso de la intercomuna de Temuco y Padre Las Casas en la Región de la Araucanía chilena, en donde se estima habitan 350.000 personas y cerca de un $90 \%$ de las emisiones totales anuales de material particulado — principal contaminante de la zona - son originadas por la combustión de leña en alrededor de 86.000 viviendas. En las referidas inter-comunas, se estima la existencia de un número total aproximado cercano a los 100.000 puntos de descarga de contaminantes provenientes de tubos de cocinas a leña, salamandras, estufas a leña, etc. (ver CONAMA, 2007a) ${ }^{1}$. Este tipo de problema se encuentra también documentado a nivel internacional en Krumdieck (2008) y Barna et al. (2002).

Desde el punto de vista de diseño de regulación orientado a mejorar la calidad del aire se trata de un desafío relevante, ya que se tiene un gran número de pequeñas fuentes de contaminación, que contribuyen al problema como consecuencia de actividades destinadas al bienestar familiar y del hogar. Debido a los altos precios relativos de los combustibles sustitutos puede suponerse que la leña seguirá siendo la principal fuente de generación energética de los hogares (Gómez-Lobo, 2005). Sin embargo, la calidad de la leña utilizada y en particular su contenido de humedad, influye de manera importante sobre el nivel de emisiones generado. Al respecto véase por ejemplo, Universidad de Concepción (2002) y CONAMA-DICTUC $(2008)$.

Los esfuerzos desplegados por las autoridades ambientales se han concentrado hasta ahora principalmente en la realización de campañas de educación sobre el correcto uso del combustible entre la ciudadanía, de manera de reducir las emisiones en la etapa de combustión. Las autoridades ambientales también han reconocido la importancia de potenciar la sustitución de leña húmeda por seca. Dicha sustitución podría ser lograda a través de incentivos económicos o mediante fiscalización sobre la misma cadena de comercialización de la leña. Estos incentivos podrían generar mayores efectos que solamente los llamados a una conducta ambientalmente responsable.

En este trabajo evaluamos entonces el uso de instrumentos económicos como parte de una política ambiental orientada a inducir mayor consumo y producción de leña seca. El análisis a desarrollar tiene un carácter pionero, dado que no existe una documentación sistemática sobre el uso y potencial efecto de incentivos económicos para el propósito específico de mejorar la calidad del combustible leña utilizado.

Exploramos mediante el uso de simulaciones numéricas la propiedad de costo-efectividad de utilizar incentivos económicos para inducir cambios en los patrones de consumo de leña por parte de los hogares localizados en zonas urbanas. Concentramos nuestro análisis al caso de las comunas de Temuco y Padre

1 Desde el año 2005 y a través del Decreto Supremo N²5, se declara zona saturada por material particulado respirable (MP-10), como concentración de 24 horas, a las comunas de Temuco y Padre las Casas. 
Las Casas, para las cuales el problema de contaminación por combustión de leña está más documentado y existe un anteproyecto de Plan de Descontaminación Atmosférica $^{2}$ (de aquí en delante PDA). Evaluamos tres sistemas de incentivos: i) subsidio a la demanda de leña seca, ii) subsidio al secado de leña orientado a pequeños productores de la misma, y iii) fiscalización para inducir cumplimiento del estándar de contenido de humedad de la leña.

Los resultados del análisis costo-efectividad sugieren que, entre las alternativas de subsidio consideradas es deseable orientar los subsidios al secado de leña por parte de productores en la etapa superior de la cadena de comercialización. Sin embargo, el impacto de los programas de subsidio sobre el nivel agregado de emisiones de material particulado y por tanto sobre la calidad ambiental es acotado. Nuestros resultados sugieren que la fiscalización para el cumplimiento es superior, en términos de costo-efectividad, a los programas de subsidio evaluados. Lo anterior es consecuencia de que la fiscalización intenta reducir directamente la proporción de leña húmeda utilizada, que es ciertamente la variable que influye en las emisiones. Además esta incidencia importante de la fiscalización puede obtenerse a un muy bajo costo para el regulador.

Este trabajo se estructura de la siguiente manera; en la Sección 2 se presenta el marco conceptual y metodológico que sirve de base para el análisis numérico del efecto de los incentivos económicos considerados para apoyar la producción, comercialización y uso de leña seca sobre el consumo de leña y las emisiones de material particulado. La Sección 3 presenta especificaciones y datos de los escenarios utilizados en la exploración de los efectos económicos y ambientales de las medidas propuestas. El análisis costo-efectividad considera el efecto simulado de la intervención sobre el consumo de leña, y consecuentemente sobre el nivel de emisiones por periodo. En la Sección 4 se presentan los resultados de las simulaciones realizadas. Finalmente, la Sección 5 recoge las principales conclusiones y recomendaciones a partir de los resultados obtenidos.

\section{InCENTIVOS ECONÓMICOS PARA Promover EL USO DE LEÑA SECA}

Los instrumentos económicos tienen como objetivo influir en las decisiones de comportamiento o elección de los agentes, al modificar los precios en los mercados sobre los que se desea intervenir. Específicamente, para el mercado de leña los incentivos económicos pueden potencialmente apoyar la producción, comercialización y uso de leña seca induciendo cambios en los precios relevantes para afectar las decisiones de demanda y/u oferta de leña seca (o los sustitutos de ésta).

El uso de leña por parte de los hogares corresponde a una decisión de demanda en la que los hogares resuelven sobre el consumo de diversos bienes; entre ellos,

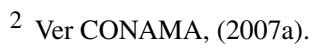


aquellos referidos a la forma de generar energía considerando los precios relevantes (precio de leña según tipos, precio de combustibles sustitutos) en conjunto con otras características de los hogares (ingresos, tamaño del grupo familiar), características de sus viviendas y equipos (tamaño de la vivienda, sistemas de aislamiento, equipos de combustión, etc.) y otras tales como restricciones de acceso ${ }^{3}$. Si una autoridad reguladora desea modificar el consumo de leña seca debiera considerar como instrumentos potenciales por el lado de la demanda aquellos que, directa o indirectamente, pueden afectar algunas de las variables determinantes de la demanda del hogar para el bien considerado. Naturalmente, juegan un rol central las variables que afecten el precio de la leña seca, así como los precios de sus sustitutos, en particular de la leña húmeda, como fuente de generación de energía.

Los incentivos económicos destinados a promover el uso de leña seca o desincentivos económicos al consumo de leña húmeda a través de su demanda, pueden considerar, entre otros, programas de transferencias específicas o subsidios a ese mercado, programas de transferencias específicas (impuestos) en el mercado de leña húmeda, y subsidios para el gasto en combustible destinado a calefacción y energía para cocción (cocina) en hogares que no participan en el mercado de leña pero que, sin embargo, utilizan leña u otros combustibles altamente contaminantes.

El consumo de leña seca puede ser también incentivado a través de políticas que afecten directamente la oferta de leña seca en el mercado (o que desincentiven la oferta de leña húmeda). En este caso los sistemas de incentivo (desincentivo) pueden operar a distintos niveles de la cadena productiva: propietarios de madera en pie, productores de trozos, intermediarios, y distribuidores. Entre los tipos de instrumentos económicos podemos incluir los siguientes: subsidio al secado de leña, incentivos tributarios a productores y/o comercializadores de leña seca y, financiamiento de corto plazo para capital de trabajo (destinado a cubrir financiamiento requerido para iniciar anticipadamente la producción de cada temporada).

Adicionalmente, es interesante destacar que en el caso de las comunas de Temuco y Padre Las Casas, existe una norma considerada en el PDA que prohíbe el uso y comercialización de leña húmeda. Incentivos económicos al cumplimiento a través de presión fiscalizadora (vigilancia y sanciones económicas) pueden ser considerados también como herramientas para promover el uso de leña seca.

Luego de analizar detalladamente la información disponible sobre la cadena producción-comercialización de la leña para el estudio de caso particular en Temuco y Padre Las Casas, se consideran, inicialmente, dos esquemas de subsidios: subsidio a la demanda de leña seca y subsidio a productores de leña que abastecen el mercado en estudio. En el primer caso, el subsidio está destinado a cubrir parcialmente la brecha de precios existente entre leña seca y leña húmeda.

3 Evidencia empírica respecto a la relevancia de estos factores en la elección de combustibles para generación de energía de parte de hogares urbanos ha sido recientemente documentada en Barnes, 'Krutilla y Hyde (2005)', ver especialmente el Capítulo 3. Sin embargo, al menos para el caso urbano, no se reportan en general en la literatura modelos que describan el comportamiento específico de los hogares en cuanto a elección de combustibles, incluyendo la leña, en dependencia de los factores mencionados (Ver por ejemplo, Heltberg, 2005 y Heltberg et al., 2000). 
En el segundo caso, el subsidio está destinado a cubrir los costos de secado. Adicionalmente, consideramos también una evaluación de costo-efectividad de las medidas destinadas a desarrollar fiscalización para inducir cumplimiento de la norma de contenido de humedad en la leña ${ }^{4}$.

Estudiamos el efecto de las medidas propuestas desde las siguientes perspectivas: proporción de leña seca y húmeda consumida, costos asociados por parte del regulador (financiamiento requerido) y el impacto esperado en términos de reducción de emisiones. La combinación de nuestro análisis de costo en conjunto con el impacto sobre emisiones, permite obtener indicadores de costo-efectividad.

\subsection{Subsidio a la Demanda de Leña Seca}

El esquema conceptual considera un programa de subsidio de monto fijo al consumo de leña seca. La demanda y oferta de leña seca se representan como $q^{d}\left(p^{d}\right)$ y $q^{s}\left(p^{s}\right)$, donde $q^{d}$ representa cantidad demandada, $q^{s}$ es la cantidad ofrecida y $p^{d}$ y $p^{s}$ son los precios por unidad de leña seca, respectivamente ${ }^{5}$. Un subsidio de monto fijo $(s)$ introduce una brecha entre el precio que paga el demandante $p^{d}$ y el precio que recibe el oferente $p^{s}$,

(1) $\quad p^{s}-p^{d}=s$

Utilizando variaciones "pequeñas" $(\Delta)$ para examinar el impacto de cambios en $s$ se tiene,

$$
\Delta p^{s}-\Delta p^{d}=\Delta s
$$

En equilibrio de mercado se requiere:

$$
\Delta q^{d}=\Delta q^{s}
$$

Es posible mostrar que un subsidio de monto fijo que modifique los precios relevantes para demandantes (y quizás también el de oferentes) de leña seca, reducirá el precio pagado por los consumidores, incrementará el precio recibido por los productores/comercializadores, e incrementará el consumo de leña seca (reduciendo de paso el consumo de leña húmeda y potencialmente también el de otros combustibles sustitutos).

4 La elección de los instrumentos económicos considerados en el trabajo ha estado guiada por dos factores básicos. Primero, el análisis de la información disponible y las medidas regulatorias actualmente propuestas en este ámbito. Segundo, la viabilidad de los instrumentos y su factibilidad de aplicación práctica.

5 Naturalmente, la demanda de leña seca o húmeda no depende solamente del precio o de variables puramente económicas. Por ejemplo, la leña húmeda, a pesar de presentar desventajas tanto en términos de nivel de emisiones como también para el encendido, podría ser preferida a iguales condiciones, debido a que se consume más lentamente. 
La magnitud de los efectos sobre los precios antes indicados, se puede aproximar mediante las siguientes expresiones (ver por ejemplo, Katzner, $198 \overline{8}$ y Nicholson, 1997):

$$
\begin{aligned}
& \frac{\Delta p^{d}}{\Delta s}=\frac{\eta^{s}}{\eta^{d}-\eta^{s}}<0 \\
& \frac{\Delta p^{s}}{\Delta s}=\frac{\eta^{d}}{\eta^{d}-\eta^{s}}>0
\end{aligned}
$$

Donde $\eta^{d}$ y $\eta^{s}$ representan elasticidad de demanda y oferta, respectivamente. Las ecuaciones (4) y (5) sugieren que el impacto de un subsidio en el mercado de la leña seca producirá efectos sobre los precios relevantes para los hogares consumidores y los productores/comercializadores ${ }^{6}$. La cuantificación del efecto del subsidio sobre el precio de demanda de leña seca permite calcular el impacto de éste sobre la cantidad consumida de leña seca, lo cual puede ser realizado usando la siguiente expresión:

$$
\Delta \% q^{d}=\eta^{d} \times \Delta \% p^{d}>0
$$

La expresión anterior puede reescribirse en términos de la variación absoluta en la cantidad de leña seca demandada, como

$$
\Delta q^{d}=\frac{\eta^{d} s q^{d}}{p^{d}}
$$

Bajo el supuesto que el aumento en consumo de leña seca equivale a una reducción de igual magnitud en el consumo de leña húmeda, y que el total de leña consumida se mantiene constante, es posible calcular el impacto del subsidio sobre la proporción de leña seca/húmeda consumida.

Considerando diferentes escenarios y/o programas de subsidios, el costo de cada programa específico, puede calcularse como el producto entre el subsidio por unidad de leña, $s$, y el monto total de leña seca consumido, según se muestra en la siguiente expresión:

$$
G_{d}=s\left(q^{d}+\Delta q^{d}\right)=s\left(q^{d}+\frac{\eta^{d} s q^{d}}{p^{d}}\right)>0
$$

${ }^{6}$ Las expresiones contenidas en las ecuaciones (4) y (5) son obtenidas suponiendo que los mercados de factores productivos, en este caso aquellos asociados a la producción de leña, no se ven alterados. Este análisis no considera, por tanto, posibles efectos de una intervención en el mercado del producto sobre mercados de insumos y las interacciones asociadas. El principal factor productivo necesario para producir leña seca es la leña, cuya oferta se asume perfectamente elástica, esto implicaría en nuestro caso que la cantidad total de leña utilizada se mantiene constante. Para un análisis exhaustivo del efecto de una intervención (impuesto) en un mercado específico considerando mercados de factores productivos relacionados, véase Katzner $(\overline{198})$. Notamos que esta aproximación puede también desarrollarse a partir del estudio de modelos microeconómicos de comportamiento de hogares, lo cual excede los objetivos de nuestro trabajo. En cualquier caso, la agregación de la demanda individual de hogares desembocará en un estudio similar al anterior que se base en la demanda de mercado. 
Dado el efecto de los programas de subsidio sobre el consumo de leña seca, se puede simular el nivel de global de emisiones de material particulado conocido como MP-10 — de aquí en más $e$ - inducido por la reducción en el precio de la leña seca.

$$
\Delta e=e\left(q^{d}+\Delta q^{d}\right)-e\left(q^{d}\right)<0
$$

El signo de la ecuación (9) es negativo debido a que se cuantifican las emisiones asociadas tanto al consumo de leña seca como de leña húmeda. Dado que el consumo total de leña se supone constante, al aumentar el consumo de leña seca disminuye el consumo de leña húmeda. Como la combustión de leña seca genera un nivel de emisiones menor que aquel asociado a la combustión de leña húmeda, entonces las emisiones globales $e$ disminuyen al aumentar el consumo de leña seca $q^{d}$. Finalmente, combinando información de gasto agregado del programa de incentivos económicos en la ecuación (8), en conjunto con el nivel de emisiones generado en cada escenario en la ecuación (9), pueden ser obtenidas medidas de costo-efectividad. Si las emisiones agregadas dependen linealmente de la cantidad de leña seca consumida, entonces la ecuación (9) se reduce a:

$$
\Delta e=-\beta \Delta q^{d}<0
$$

Donde $\beta$ representa la diferencia en el coeficiente de emisión entre leña húmeda y leña seca. En este caso lineal, el indicador de costo-efectividad es una función también lineal del monto de subsidio y toma la siguiente forma:

$$
C E_{d}=-\frac{1}{\beta}\left(\frac{p^{d}}{\eta^{d}}+s\right)
$$

El indicador de costo-efectividad en la ecuación (11) fue calculado como el cuociente entre el gasto en subsidio dado por la ecuación (8) y la reducción en las emisiones de material particulado logradas reflejado en la ecuación (10). Esta magnitud cuantifica el gasto en subsidio a la demanda por cada tonelada reducida de material particulado.

\subsection{Subsidio a los Productores de Leña Seca}

El análisis respecto al efecto de incentivos económicos orientados a la oferta de leña seca considera un subsidio al secado de leña. Este subsidio se considerará orientado a financiar costos de oportunidad que provienen del retraso en la venta producido por la espera a que concluya el secado de la leña ${ }^{7}$.

7 Otros costos posibles asociados al secado, como por ejemplo financiamiento de capital de trabajo extra requerido en la preparación anticipada de la leña a secar para toda la temporada o infraestructura básica para el secado, no son considerados en esta evaluación. 
Basados en OOtero et al. (2004), la metodología utilizada para calcular el costo de oportunidad asociado al secado supone que se conoce el precio de venta de la leña húmeda en la transacción entre productores y comercializadores, y la tasa de interés mensual que observaría un productor genérico. Con esta información, el costo de oportunidad de secar la leña se estima como el retorno por concepto de intereses que se genera al depositar un monto igual al precio de la leña durante el tiempo de secado requerido a la tasa supuesta. Se necesitan entonces tres datos básicos: el tiempo de secado, el precio de venta de la leña húmeda al nivel de los productores y la tasa de interés mensual observada por los mismos ${ }^{8}$.

El costo total de este tipo de subsidio, será el producto entre las cantidades anuales requeridas de leña para cada estimación de consumo considerada y el costo de secado por unidad $(\rho)$ para cada escenario propuesto.

$$
G_{s}=\rho\left(q^{d}+\Delta q^{d}\right)
$$

El cálculo de indicadores de costo efectividad para el subsidio del secado orientado a los productores, estará determinado por el cuociente entre los costos totales de este subsidio mostrado en la ecuación (12) y los niveles de reducción de emisiones previamente calculados. Si comparamos niveles de reducción de emisiones iguales a los inducidos a través del subsidio a la demanda en la ecuación (10), el indicador de costo-efectividad en este caso toma la forma:

$$
C E_{s}=\frac{\rho}{s}\left(-\frac{1}{\beta}\left(\frac{p^{d}}{\eta^{d}}+s\right)\right)
$$

Esta ecuación puede interpretarse como el gasto en subsidio al secado de leña por tonelada de emisiones reducida. Puede notarse que el análisis comparativo del indicador de costo-efectividad de los dos instrumentos de subsidio mostrado en las ecuaciones (13) y (11) depende solamente de la relación entre $\rho$ y $s$, pero no de la elasticidad $\eta^{d}$.

\subsection{Fiscalización para Inducir Cumplimiento de la Norma de Contenido de Humedad}

Un componente fundamental de cualquier medida de regulación aplicada será la existencia de un esfuerzo de fiscalización acompañante a las normas

${ }^{8}$ En el contexto de nuestro trabajo, los precios de la leña húmeda y seca en la ciudad son observados y fijos, y naturalmente sujetos a diversos factores determinantes, uno de los cuales es el costo de oportunidad del secado en zonas rurales. La diferencia de precios entre leña seca y leña húmeda en la zona urbana se fija posteriormente en \$1.500. Esto no significa que el costo de oportunidad de secado en el campo quede determinado también en ese monto. En este sentido, la tasa está fija al extender el periodo de secado, pero naturalmente el costo de oportunidad es mayor al extenderse tal periodo. En todas las simulaciones presentadas, el costo de oportunidad asociado al secado de leña en la zona rural no excede la diferencia de precios entre leña seca y húmeda observado en la zona urbana. 
establecidas, en este caso en cuanto a la prohibición de la comercialización de leña húmeda en ambas comunas (ver CONAMA, 2007a) ${ }^{9}$. Esto traería consigo un incremento en el precio efectivo de comercializar y/o consumir leña húmeda (producto de las probabilidades de ser fiscalizado). Esta es la otra vía para reducir el diferencial de precios existente entre leña seca y leña húmeda: incrementando el valor de la leña húmeda, dado que los comercializadores de ésta incorporarían en sus costos, la sanción esperada de ser sorprendidos incumpliendo la normativa, lo cual automáticamente incentivaría la oferta de leña seca.

El análisis económico estándar de la fiscalización y cumplimiento tiene como punto de partida el trabajo de Becker (1968). En general, siguiendo el enfoque de Becker, un agente que está decidiendo respecto a transgredir una norma o regulación, compara el costo esperado de una infracción con el beneficio esperado de ésta. El costo esperado de cometer una infracción a la regulación está dado por la sanción esperada, la que a su vez está determinada por la frecuencia de la actividad de monitoreo o vigilancia de la agencia reguladora y la estructura de las sanciones dada por la propia regulación o el sistema judicial. El beneficio de infringir una regulación está representado por las ganancias económicas derivadas de la actividad prohibida o regulada ${ }^{10}$.

Consideramos a continuación un modelo de un comercializador individual de leña que enfrenta un estándar respecto al contenido de humedad de la leña. El modelo conceptual sirve de base para las simulaciones numéricas que desarrollamos en las siguientes secciones. La producción de leña se realiza en zonas rurales, y debe por tanto ser transportada a los centros urbanos para su comercialización. Sea $\delta$ el porcentaje de leña que se transporta húmeda, $0 \leq \delta \leq 1$. En nuestro modelo la proporción de leña que se consume húmeda coincide con $\delta$. Es por eso que este parámetro también expresa el nivel de cumplimiento de la regulación que prohíbe el consumo y comercialización de leña húmeda. Suponemos que, debido a problemas de información, el agente puede vender un porcentaje de la leña húmeda como leña seca ${ }^{11}$. Denotamos tal fracción como $d(\delta)$, donde $0 \leq d(\delta) \leq 1$ y $d^{\prime}(\delta) \leq 0$, para una discusión sobre este parámetro y las hipótesis asociadas ver Apéndice. Adicionalmente, denotamos el nivel de precio de leña seca y húmeda en la zona urbana como $P_{U S}$ y $P_{U H}$, respectivamente, con $P_{U S} \geq P_{U H}$. De manera análoga $P_{R S}$ y $P_{R H}$ representan el precio de leña

9 Entendemos por fiscalización el conjunto de acciones destinadas a inducir cumplimiento de los agentes bajo regulación. Dichas acciones se relacionan con la utilización de dos instrumentos: vigilancia o inspecciones con el fin de detectar infracciones, y la aplicación de sanciones en el caso que una trasgresión ser descubierta. Otras actividades posibles de desarrollar para inducir cumplimiento incluyen negociación o contacto directo con los agentes regulados, transferencia de conocimiento, transferencia de tecnología, etc.

10 En un sistema de regulación basado en políticas ambientales de control directo, por ejemplo una norma sobre contenido de humedad de la leña, una fuente regulada (por ejemplo un productor, o comercializador) está en infracción si el nivel de contenido de humedad en la leña excede el estándar.

11 Como hemos señalado anteriormente, la calidad de la leña ofrecida y consumida en la zona es heterogénea en cuanto a su contenido de humedad. En particular esta heterogeneidad impide una comercialización completamente transparente debido a la presencia de asimetría de información, así como también a diferencias importantes en las unidades de medida. 
seca y húmeda en la zona rural, respectivamente, con $P_{R S} \geq P_{R H}$. En nuestro modelo la diferencia de precios entre leña seca y húmeda en la zona rural estará asociada solamente al costo de secado analizado en la sección previa, i. e. $\rho=P_{R S}-P_{R H}$. Suponemos que el comercializador considera los precios como dados en su decisión.

En presencia de fiscalización para inducir cumplimiento de la norma de prohibición de transporte y comercialización de leña húmeda, el beneficio esperado por unidad física de leña, de un comercializador neutral al riesgo, es una función del porcentaje de leña húmeda transportada desde la zona rural a la zona urbana e incluye cuatro componentes cómo se puede ver en la ecuación (14): el beneficio asociado a la comercialización de leña húmeda como tal, el beneficio por comercialización de leña húmeda como leña seca, el beneficio que se obtiene al comercializar leña seca, y la sanción esperada por infringir la regulación. Escribimos este beneficio esperado $(B)$ como:

$$
\begin{aligned}
B(\delta)= & \left(P_{U H}-P_{R H}\right)(1-d(\delta)) \delta+\left(P_{U S}-P_{R H}\right) d(\delta) \delta \\
& +\left(P_{U S}-P_{R S}\right)(1-\delta)-\pi \delta \phi
\end{aligned}
$$

En nuestro modelo los costos de transporte dependen sólo del monto total de leña (y no de su calidad). Por ende, estos no influyen en el porcentaje de leña húmeda comercializada y han sido excluidos en nuestro análisis ${ }^{12}$. La sanción esperada por unidad se obtiene multiplicando la probabilidad de ser auditado, que denotamos $\pi$, por el porcentaje de leña húmeda transportada, y por el monto de sanción por unidad física de leña húmeda, $\phi$.

Luego de alguna manipulación, es posible mostrar que la expresión en la ecuación (14) puede escribirse también como:

$$
B(\delta)=\left(P_{U S}-P_{R S}\right)+\delta\left(\begin{array}{l}
\left(P_{U S}-P_{U H}\right) d(\delta) \\
+\left(\left(P_{U H}-P_{R H}\right)-\left(P_{U S}-P_{R S}\right)\right)
\end{array}\right)-\pi \delta \phi
$$

El porcentaje de leña húmeda comercializado es aquel que maximiza la ecuación (15). Suponiendo que la función de beneficios es estrictamente cóncava en $\delta$, la condición necesaria y suficiente para el beneficio máximo del intermediario está dada por:

$$
\begin{aligned}
B^{\prime}(\delta)= & \left(P_{U S}-P_{U H}\right) d(\delta)+\left(\left(P_{U H}-P_{R H}\right)-\left(P_{U S}-P_{R S}\right)\right) \\
& +\delta\left(P_{U S}-P_{U H}\right) d^{\prime}(\delta)-\pi \phi=0
\end{aligned}
$$

12 Ciertamente la leña húmeda al ser más pesada podría inducir un mayor costo de transporte. Para mantener el modelo manejable, hemos excluido el análisis del referido efecto. 
En ausencia de fiscalización $(\pi=0)$, lo cual constituye la situación base, usando la ecuación (16), el nivel óptimo está dado por $\delta=\bar{\delta}$, donde $0<\bar{\delta}<1$ se define como:

$$
\bar{\delta}=\frac{\left[\left(P_{U S}-P_{U H}\right)-\left(P_{R S}-P_{R H}\right)\right]-d(\bar{\delta})\left(P_{U S}-P_{U H}\right)}{\left(P_{U S}-P_{U H}\right) d^{\prime}(\bar{\delta})}
$$

En el Apéndice exploramos cierta estructura de la función $d(\delta)$ que garantiza la factibilidad de la situación base.

Luego de ajustar el modelo anterior, la ecuación (16) permite determinar para cada valor de la proporción de leña húmeda transportada $\delta$, un nivel de fiscalización — expresado mediante la probabilidad de ser auditado $\pi(\delta)$ — asociado, que induce en el comercializador la selección de la proporción $\delta$ mediante la fiscalización.

Para nuestro análisis suponemos que a partir de la situación base se desea inducir un nuevo escenario en el cual la proporción de leña húmeda transportada disminuya hasta un valor prefijado. El análisis anteriormente mencionado proporciona un nivel de esfuerzo de fiscalización (probabilidad de detección) $\pi$ que debe ser implementado por el regulador.

Las acciones destinadas a inducir cumplimiento demandan el uso de recursos y por lo tanto tienen costos asociados. En particular, los costos de fiscalización $(C F)$ están determinados por la tecnología de monitoreo o vigilancia utilizada (forma en que se realizan las inspecciones, recursos utilizados, personal, vehículos, etc.), el esfuerzo de vigilancia desplegado (por ejemplo frecuencia y cobertura de las inspecciones) el poder disuasivo de las sanciones (por ejemplo, sanciones altas pueden ahorrar recursos en términos de esfuerzo de vigilancia manteniendo igual poder disuasivo). Por último, dado un nivel de cumplimiento deseado, el costo de fiscalizar para inducir cumplimiento estará también determinado por el tamaño de la población regulada.

Suponiendo que la imposición de sanciones no genera costos y considerando que las sanciones están determinadas exógenamente, esto es, su nivel no depende de la autoridad fiscalizadora, el costo de fiscalización $(C F)$ está determinado por el costo de vigilancia o inspección y el número de inspecciones que es necesario realizar por periodo. Es importante destacar que el número de inspecciones que se debe realizar, estará determinado por el nivel de cumplimiento (alternativamente, incidencia de incumplimiento o infracciones) de la regulación.

Sea $\psi$ el costo de mantener un equipo de fiscalización o control, $N$ el universo de inspecciones a realizar y $n$ el número de controles o auditorías que un equipo puede realizar ${ }^{13}$. De esta forma, el costo de lograr un determinado nivel de cumplimiento de la regulación $\delta$, puede escribirse como:

$$
C F(\delta)=\psi \times\left(\frac{N}{n}\right) \times \pi(\delta)
$$

13 Con la notación introducida el número de equipos de fiscalización necesarios para controlar al total de la población regulada estaría dado por $\frac{N}{n}$. 
Un cambio regulatorio que incluya, por ejemplo, medidas más restrictivas (valores más pequeños de $\delta$ ) generará incremento en los costos de fiscalización. En la ecuación (18) ello se asocia a un incremento en la probabilidad de auditoría $\pi(\delta)$.

Si tomamos como base un nivel de cumplimiento $\delta$ que genere una reducción de emisiones idéntica a la inducida a través del subsidio a la demanda en la ecuación (10), el indicador de costo-efectividad, entendido en este caso como el cuociente entre los costos de fiscalización y la reducción de emisiones lograda, toma la siguiente forma luego de cierta manipulación algebraica:

$$
C E_{F}=-\psi \frac{N}{n}\left[\frac{\rho}{\beta q^{h} \phi}\right]
$$

Donde $q^{h}$ es la cantidad de leña húmeda consumida en la situación base. Nótese que el coeficiente de costo-efectividad no depende del nivel de cumplimiento $(\delta)$ perseguido. Como es de esperar, el coeficiente costo-efectividad mejora (se reduce) si aumenta la sanción $\phi$. Esta mejoría es solamente viable en la medida en que el nivel de la multa necesaria sea susceptible de ser impuesta. Naturalmente, existe un máximo implementable para $\phi$.

Finalmente, como se observa en la ecuación (19) el coeficiente costo-efectividad empeora (se incrementa) junto con el costo de secado de la leña, $\rho$. Esto puede interpretarse como que, un mayor diferencial de precios entre leña seca y húmeda en la zona rural incentiva el transporte de leña húmeda, lo cual se traduce en la necesidad de mayor esfuerzo de auditoría y por ende de mayores costos de fiscalización para alcanzar el mismo nivel de cumplimiento.

\section{ESPECIFICACIONES, DATOS Y EsCENARIOS}

Los antecedentes conocidos sobre el funcionamiento del mercado de leña en Temuco y Padre Las Casas, describen básicamente la participación de tres agentes económicos: productores, distribuidores y consumidores ${ }^{14}$.

La oferta de leña está caracterizada por un gran número (varios cientos) de pequeños productores localizados en las comunas rurales vecinas a la capital de la Región de la Araucanía, y su cadena de comercialización (distribución) en las zonas urbanas principalmente bajo dos instancias, la venta en camiones y el acopio y venta en leñerías. La demanda de leña por su parte, se caracteriza por la preferencia en el uso de la leña de los consumidores de las comunas en estudio, para calefacción y cocción de alimentos, en relación a otros combustibles (ver, por ejemplo, UDEC, 2002; CONAMA-DICTUC, 2008).

14 Véase por ejemplo, Universidad de Concepción $(20 \overline{2})$, Ötero et al. $(200 \overline{4})$. Los autores agradecen además detallada y valiosa información sobre el funcionamiento del mercado de leña en Temuco y Padre Las Casas provista por Mauricio Lobos, Conama-Región de la Araucanía, a través de contacto personal. 
El escenario base de nuestro análisis caracteriza el consumo de leña, las emisiones y los precios $\left(\$ / \mathrm{m}^{3}\right.$ estéreo) en las comunas de Temuco y Padre Las Casas $^{15}$. Para estimar el consumo anual de leña, se consideró el número de viviendas y el tipo de artefacto empleado para calefacción. Para el año 2008 se obtiene un total de 87.870 viviendas con un $13 \%$, 34\% y $53 \%$ de las mismas pertenecientes a los niveles socioeconómicos alto, medio y bajo respectivamente. Los tipos de artefactos considerados fueron cocina, salamandra, estufa simple, estufa doble cámara y chimenea. Como base para calcular el número de artefactos utilizados en las comunas y el estimado de su consumo anual, se tomaron las probabilidades de uso de cada artefacto según nivel socioeconómico, de acuerdo a lo propuesto en el análisis de Sanhueza et al. se estima el consumo total de leña en $496.587 \mathrm{~m}^{3}$ estéreo anuales.

Adicionalmente, hemos considerado para el escenario base que el consumo de leña seca alcanza a un $20 \%$ del total de consumo en base anual, lo cual es un estimado encontrado en la literatura revisada (ver, por ejemplo, Sanhueza et a $\bar{l}_{\text {. }}$, 2006), alcanzando los $99.317 \mathrm{~m}^{3}$ estéreo. El $80 \%$ restante de la leña consumida es leña húmeda (397.270 $\mathrm{m}^{3}$ estéreo). Esta estimación acerca de las proporciones leña seca/leña húmeda utilizada en el escenario base si bien difieren de las utilizadas en el inventario de emisiones más reciente (CONAMA-DICTC, 2008), coinciden con las propuestas en estudios anteriores (por ejemplo, Sanhueza et al., 2006). Cabe señalar que varias de las estimaciones de esta proporción (por ejemplo las de CONAMA-DICTUC, 2008) no están obtenidas a partir de mediciones directas del contenido de humedad de la leña ${ }^{16}$.

Para estimar las emisiones se tomó el consumo calculado ya estratificado en cuanto a nivel socio económico, artefacto utilizado y tipo de leña (seca o húmeda), y se convirtió a unidades de masa en base anual (kg/año). Estos valores se multiplicaron por los correspondientes estimados de los factores de emisión por equipo y tipo de leña. En nuestro caso se obtiene para el escenario base una estimación de las emisiones anuales totales del orden de 3.351 toneladas ${ }^{17}$.

El modelo utilizado para el cálculo de las emisiones no muestra, desde un punto de vista conceptual, ningún indicio que sustente la hipótesis de linealidad enunciada en la ecuación (10). No obstante, los resultados de las simulaciones nu-

15 Las estimaciones para el cálculo del consumo de leña y emisiones se basaron en los datos y modelo de Sanhueza (2006). La unidad de medida denominada $\mathrm{m}^{3}$ estéreo es frecuentemente utilizado en el caso de la leña. Para ver su definición y relación con otras unidades puede consultarse, por ejemplo Navarro $(2005)$.

16 En nuestro conocimiento, un caso en que las estimaciones de la proporción leña seca/leña húmeda utilizada se obtienen a partir de mediciones directas es el estudio Universidad de Concepción (2002).

17 Existe un inventario de emisiones más reciente (ver CONAMA-DICTUC, 2008) que utiliza otro modelo y otros valores para algunos parámetros. Esto varía los resultados de la estimación base. Por ejemplo el nivel de emisiones de material particulado en Temuco y Padre Las Casas asociado a la combustión de leña de parte de hogares durante el año 2005 se estima en 5.374,4 toneladas. Esta cifra es significativamente superior a la que caracteriza nuestro escenario base. Se evaluó la posibilidad de usar, para efecto de corridas de simulaciones, el modelo de este inventario reciente o los parámetros del mismo. Sin embargo, desechamos tal análisis debido a que éste modelo no ofrece suficiente flexibilidad con relación al contenido de humedad utilizada por los hogares de las comunas bajo estudio. Por otra parte nuestro análisis se centra en las variaciones relativas y no en la exactitud de los valores base. 
méricas sugieren la existencia de esta dependencia lineal (ver Sección 4.1 y Cuadro 5) aunque el valor específico de $\beta$ no puede ser calculado directamente ${ }^{18}$.

Finalmente los precios base han sido definidos de acuerdo a la información provista por CONAMA, Región de la Araucanía, a partir de los registros sobre reporte de contenido de humedad de la leña en Temuco y Padre Las Casas, para los años 2004-2007 (CONAMA,2007 los precios promedio observados para el periodo, que alcanzan a $\$ 16.500 / \mathrm{m}^{3}$ estéreo para leña seca y $\$ 15.000 / \mathrm{m}^{3}$ estéreo para leña húmeda. Para tener una idea del impacto en el gasto asociado al consumo de un tipo de leña o de otro (húmeda/seca), considere que un hogar de bajos ingresos que consuma alrededor de $11 \mathrm{~m}^{3}$ estéreo de leña anualmente, vería incrementado el porcentaje de su ingreso anual que destina al consumo de leña en 1,3 puntos porcentuales al cambiarse desde leña húmeda hacia leña seca. El mismo análisis arroja una variación de 0,4 y 0,1 puntos porcentuales en el caso de hogares de ingresos medios y bajos, respectivamente ${ }^{19}$.

Acorde con la metodología descrita en la sección previa, el análisis numérico requiere información respecto a elasticidades. Notamos que, aunque la demanda total de leña podría ser relativamente inelástica, nuestro interés se concentra en el impacto de cambios de precio sobre el consumo de leña seca. En nuestra revisión de literatura publicada, no encontramos estimaciones de elasticidades de demanda y oferta para la leña seca ni a nivel nacional ni internacional.

En ausencia de información de elasticidades tanto de la demanda como de la oferta de leña seca, es conceptualmente factible para los efectos de análisis de sensibilidad numérico, fijar una de ellas y considerar distintos escenarios para la otra. En nuestro caso, hemos considerado una elasticidad de oferta infinita $\left(\eta^{s}=\infty\right)$ y tres valores alternativos para la elasticidad precio de la demanda de

18 Desde el punto de vista teórico, la linealidad de las emisiones agregadas con respecto a la cantidad de leña seca consumida es sólo un supuesto que simplifica las expresiones analíticas, pero evidentemente cuestionable, como ha observado uno de los evaluadores de Cuadernos de Economía. Entre los objetivos de nuestro trabajo no se incluye la modelación detallada para el cálculo de emisiones, la cual probablemente, conduzca a un comportamiento no lineal. La herramienta de simulación para el cálculo de emisiones está fijada a priori, y ha sido utilizada y validada por la Comisión Nacional del Medio Ambiente-Región de La Araucanía (Sanhueza, 2006). Un análisis inicial no permite intuir a priori un comportamiento lineal, sin embargo, nuestros resultados presentan evidencia numérica de linealidad, lo cual invita a trabajar con esa hipótesis. Las razones por las cuales el método de cálculo resulta ser lineal son diversas y su estudio detallado está fuera del alcance de nuestro trabajo. No obstante, pensamos que la linealidad resultante puede atribuirse esencialmente a la forma de cálculo de las emisiones individuales de cada hogar, las cuales están basadas en factores de emisión. Esta metodología de factores de emisiones es estándar y permite trabajar y calcular tendencias "agregadas" evitando una modelación más detallada.

19 Este análisis está basado en la información disponible en la encuesta CASEN 2006 sobre niveles de ingreso y consumo promedio de leña, así como en la información de precios promedio observados para leña seca y húmeda de la provista por CONAMA, Región de la Araucanía. Los niveles de ingreso utilizados son 102 UF anuales, 326 UF anuales, y 1265 UF anuales, respectivamente, para hogares clasificados como "bajo", "medio" y "alto". En el caso de un consumo de $11 \mathrm{~m}^{3}$ anuales de sólo leña seca, el porcentaje de ingreso anual destinado a gasto en leña asciende a 8\%, 2,5\%, y 0,7\%, respectivamente para hogares de ingreso "bajo", "medio" y "alto". 
leña seca. Los valores considerados son: demanda elástica $\left(\eta^{d}=-4\right)$, demanda de elasticidad unitaria $\left(\eta^{d}=-1\right)$ y demanda inelástica $\left(\eta^{d}=-0,3\right)$. Con el objeto de disponer de un patrón general de referencia, considérese que a nivel internacional la elasticidad precio propia estimada de la demanda de leña para combustión de parte de hogares fluctúa entre $-0,11$ y $-1,47$ (Cooke et al., 2008) ${ }^{20}$.

A partir del supuesto sobre la elasticidad de la oferta $\left(\eta^{s}=\infty\right)$ se obtiene que el impacto del subsidio se transmite completamente al precio que paga el demandante de leña seca (esto es $\Delta p^{d} / \Delta s=-1$ en la ecuación (4), en tanto que $\Delta p^{s} / \Delta s=0$ en la ecuación 5). Nótese que cualquier traspaso del subsidio al precio del oferente debido a una oferta relativamente inelástica $\left(\eta^{s} \neq \infty\right)$ puede replicarse en el caso considerado a través de una alteración correspondiente de la elasticidad de la demanda.

El análisis para el caso de subsidio a la demanda considera un programa con distintos montos destinado a cubrir parcialmente la brecha de precio existente entre leña seca y leña húmeda ( $\$ 1.500$ por $\mathrm{m}^{3}$ estéreo). Así por ejemplo, y según se presenta en detalle en el Cuadro 1, un subsidio cuyo monto corresponde al $60 \%$ de la diferencia de precio entre leña seca y leña húmeda ( $\$ 900$ por $\mathrm{m}^{3}$ estéreo), produciría una reducción en el precio de leña seca de 5,5\%, alcanzando el precio de leña seca en tal escenario un nivel de $\$ 15.600$ por $\mathrm{m}^{3}$ estéreo (ver Cuadro 1 , columna 3 , última fila).

\section{CUADRO 1}

EFECTOS DE SUBSIDIOS A LA DEMANDA SOBRE EL PRECIO DE LEÑA SECA EN TEMUCO Y PADRE LAS CASAS USADOS EN SIMULACIONES

\begin{tabular}{lccc}
\hline \multirow{2}{*}{ Efectos sobre el precio } & \multicolumn{3}{c}{$\begin{array}{c}\text { Escenarios de subsidio } \\
\text { al diferencial de precios }\end{array}$} \\
\cline { 2 - 4 } & $\mathrm{I}$ & $\mathrm{II}$ & $\mathrm{III}$ \\
\hline \% de brecha de precios cubierto por el subsidio & 30 & 60 & 90 \\
Monto del subsidio $\left(\$ / \mathrm{m}^{3}\right.$ estéreo) & 450 & 900 & 1.350 \\
$\%$ reducción precio leña seca & $-2,73$ & $-5,45$ & $-8,18$ \\
Precio leña seca con subsidio $\left(\$ / \mathrm{m}^{3}\right.$ estéreo) & 16.050 & 15.600 & 15.150 \\
\hline
\end{tabular}

Fuente: Elaboración propia.

\footnotetext{
20 Lamentablemente, no existen datos disponibles sobre consumo de leña, precios, ingreso, y otros relevantes, que permitan utilizar métodos econométricos para calibrar, por ejemplo, la elasticidad de la demanda de leña seca. Notamos, sin embargo, que el rango para la elasticidad precio de la demanda de leña seca utilizado en nuestro análisis es suficientemente amplio como para cubrir el valor real. Adicionalmente, los resultados generales de los ejercicios de simulación que se presentan en la siguiente sección no dependen del valor puntual que tome la elasticidad precio $\eta^{d}$. Finalmente, con relación a los restantes parámetros utilizados para calibrar el modelo, como por ejemplo, costos de secado, o costos de equipos de fiscalización; éstos fueron recopilados desde distintas fuentes en el marco de este trabajo.
} 
Para el análisis del impacto de subsidios sobre la oferta de leña seca, consideramos el subsidio del secado solamente en el eslabón de los productores, teniendo en cuenta que los comercializadores constituyen un mercado mayormente informal (ver Universidad de Concepción, 2002 y Otero et al., 2004). Cabe suponer que un subsidio orientado a este eslabón conlleve una formalización que implicaría mayores aumentos de precio ya no asociados exclusivamente al secado. En nuestro análisis supondremos que si se incentiva la producción de leña seca a través de los productores, el efecto sobre la producción de este tipo de leña se reflejará completamente en la cantidad de leña seca comercializada.

La información disponible sobre el tiempo de secado proviene del estudio de Navarro et al., 2005. En dicho estudio, bajo ciertas condiciones específicas de almacenamiento y especie, el tiempo de secado se estima en seis meses. Sin embargo, si las condiciones óptimas de secado no están dadas, el tiempo necesario para secar la leña puede extenderse mucho más allá de los seis meses. Con el propósito de tener en cuenta esta incertidumbre, en nuestro análisis se considera un período de secado más prolongado que asciende a 12 meses $^{21}$. Adicionalmente, la tasa de interés realizable por los productores se ha considerado en $1,4 \%$ mensual (Otero et al.

Como el precio de la leña húmeda a nivel de productor es desconocido, hemos elegido dos escenarios posibles. Estos se diferencian en el porcentaje que corresponde al productor respecto del precio final observado de la leña húmeda en las comunas de Temuco y Padre Las Casas. Se supuso entonces que el precio de la leña húmeda al que vende el productor en la zona rural puede ser de $20 \%$ y $50 \%$ de aquel observado en la zona urbana ${ }^{22}$.

Existen aspectos prácticos que complican la implementación de un subsidio del secado de leña orientado a los productores. El primero dice relación con el hecho que los productores en su mayoría seguramente no abastecen exclusivamente a las comunas de Temuco y Padre Las Casas. Este efecto sólo podría evitarse con un esfuerzo de control que resulta poco realizable. Un segundo aspecto es la dificultad de subsidiar solamente el incremento respecto a lo que hoy en día ya se seca; esto porque cualquier subsidio al secado de leña sería primeramente utilizado para financiar el secado actual y solo después de sobrepasar ese umbral se apreciaría un efecto sobre la oferta.

Teóricamente se podría subvencionar una parte del costo de secado y apostar a que la disminución en los costos de producción de leña seca obtenida, resulte en un aumento de la oferta de acuerdo a cierta elasticidad. Sin embargo, a diferencia del caso de la demanda (consumo), no existen estimaciones ni observaciones sobre el precio (de los productores) que permitan simular seriamente utilizando elasticidades hipotéticas. Por esta razón, estimamos los costos totales de secado (en el eslabón de los productores) y calculamos cuánto costaría cubrirlos con un

21 Para poder estudiar la sensibilidad del resultado respecto al tiempo de secado estimado se hicieron simulaciones con periodos de secado de 6, 12, y 18 meses. Los resultados indican que el mensaje central de nuestro ejercicio es independiente del escenario elegido. Para facilitar la presentación se muestran los resultados de las simulaciones sólo para el caso de 12 meses de secado.

22 Estos porcentajes fueron avalados por especialistas de la CONAMA, Región de la Araucanía, como representativos del rango de valores posibles del precio estimado. 
subsidio. Esto dependerá naturalmente de la cantidad de leña seca que se pretenda subsidiar la cual se obtendrá de valores estimados de consumo.

Como mencionamos en la Sección 2.3, hemos supuesto que la fiscalización para inducir el cumplimiento de la norma, sobre prohibición de la comercialización de leña húmeda, se efectúa sobre el eslabón de la cadena encargado de transportar la leña desde su lugar de cosecha (rural) hacia los consumidores (urbano). Esto permite conceptualizar la fiscalización como el control del flujo de unidades de transporte de leña a la ciudad, el cual puede cuantificarse con mayor facilidad.

Para el análisis suponemos que la leña se transporta en unidades de $30 \mathrm{~m}^{3}$ estéreo lo cual resulta en un universo de fiscalización cercano a los 15.000 viajes. Se supone que el transporte ocurre en una temporada que dura 8 meses, con lo cual tenemos cerca de 1.800 viajes a fiscalizar mensualmente. Simplificando bastante la modelación suponemos que los costos de fiscalización dependen mayormente de la cantidad de equipos fiscalizadores encargados en esta tarea durante la temporada.

Cada equipo está constituido por dos personas y tiene un costo mensual de $\$ 700.000$. Hemos supuesto que un equipo es capaz de fiscalizar mensualmente cerca de 90 entradas de leña a la ciudad. Esta cifra ya incluye la incertidumbre asociada al desconocimiento de cuáles de las diferentes rutas de acceso que tiene la ciudad son utilizadas para el transporte de leña y en qué momento. Calculando los costos y auditorías para toda la temporada, tenemos que un equipo fiscalizador cuesta $\$ 5.600 .000$ y controla un total de 750 (5\%) de los 15.000 viajes a realizarse.

En el modelo de un comercializador individual de leña presentado en la Sección 2.3, se utiliza como variable de decisión el porcentaje $\delta$ de leña húmeda transportada. Esta variable también permite medir el cumplimiento de la norma a fiscalizar. Un cumplimiento perfecto significaría un valor $\delta=0$. El escenario base, utilizado también en los otros dos instrumentos económicos, presupone que la proporción de leña húmeda consumida asciende a $80 \%(\delta=0,8)$. Para alcanzar un nivel de fiscalización requerido $(\pi(\delta))$ debemos tan solo añadir tantos equipos de control o auditoría como sean necesarios, lo cual resultará en un costo de fiscalización correspondiente (ver Sección 2.3).

Con el objetivo de hacer los cálculos comparables se tomaron escenarios de niveles de cumplimiento $(\delta)$ que induzcan reducciones de emisiones idénticas a las ya consideradas para los subsidios a la demanda y la oferta.

\section{Resultados}

En esta sección presentamos los resultados de las simulaciones numéricas desglosados según el tipo de instrumento utilizado.

\subsection{Subsidio a la demanda}

Utilizando la información respecto a los montos de subsidio al consumo de leña seca en conjunto con parámetros de elasticidad, determinamos mediante la ecuación (6) el impacto en términos del incremento en el consumo de leña seca en cada 
escenario (ver Cuadro 2). Se verifica el comportamiento esperable: para demandas más inelásticas los impactos sobre el consumo causados por el subsidio se reducen. El mayor impacto calculado se obtiene con demanda elástica y subsidio de $90 \%$ del diferencial de precio (Escenario III en Cuadro 2). En dicho caso, nuestro cálculo sugiere un incremento en la cantidad consumida de leña seca de aproximadamente $33 \%$. El menor impacto calculado se observa para un programa de subsidio de $30 \%$ de brecha de precio (Escenario I, Cuadro 2) con demanda inelástica, escenario en el cual el consumo de leña seca se incrementaría en sólo un $0,8 \%$.

El incremento calculado sobre el consumo de leña seca en cada uno de los escenarios definidos permitió, bajo los supuestos previamente descritos, recalcular la proporción de leña seca sobre el consumo total de leña en las comunas de Temuco y Padre Las Casas (ver Cuadro 3). Mientras en el escenario base la proporción de leña seca consumida alcanza a $20 \%$ del total, se observa que, mientras más ambicioso es el programa de subsidio en términos de reducción de la brecha de precios, mayor impacto genera éste en términos de la proporción de leña seca consumida. Se observaron cambios significativos en la proporción de

\section{CUADRO 2 \\ CAMBIO PORCENTUAL EN CANTIDAD CONSUMIDA DE LEÑA SECA EN TEMUCO Y PADRE LAS CASAS}

\begin{tabular}{lcrc}
\hline \multirow{2}{*}{$\begin{array}{l}\text { Elasticidad } \\
\text { (en porcentaje) }\end{array}$} & \multicolumn{3}{c}{ Escenarios de subsidio al diferencial de precios } \\
\cline { 2 - 4 } & $\mathrm{I}$ & $\mathrm{II}$ & \multicolumn{1}{c}{ III } \\
\hline Demanda elástica & 10,91 & 21,82 & 32,73 \\
Elasticidad unitaria & 2,73 & 5,45 & 8,18 \\
Demanda inelástica & 0,82 & 1,64 & 2,45 \\
\hline
\end{tabular}

Fuente: Elaboración propia.

Nota: Los valores establecidos para las elasticidades son: Demanda Elástica $\left(\eta^{d}=-4\right)$, Demanda con Elasticidad Unitaria $\left(\eta^{d}=-1\right)$, Demanda Inelástica $\left(\eta^{d}=-0,3\right)$.

\section{CUADRO 3 \\ PORCENTAJE DE LEÑA SECA-LEÑA HÚMEDA CONSUMIDA PARA DISTINTOS ESCENARIOS EN TEMUCO Y PADRE LAS CASAS}

\begin{tabular}{lccc}
\hline \multirow{2}{*}{$\begin{array}{l}\text { Elasticidad } \\
\text { (en porcentaje) }\end{array}$} & \multicolumn{3}{c}{ Escenarios de subsidio al diferencial de precios } \\
\cline { 2 - 4 } & I & II & III \\
\hline Demanda elástica & $22,2-77,8$ & $24,4-75,6$ & $26,5-73,5$ \\
Elasticidad unitaria & $20,5-77,8$ & $21,1-78,9$ & $21,6-78,4$ \\
Demanda inelástica & $20,2-79,8$ & $20,3-79,7$ & $20,5-79,5$ \\
\hline
\end{tabular}

Fuente: Elaboración propia.

Nota: Los valores establecidos para las elasticidades son: Demanda Elástica $\left(\eta^{d}=-4\right)$, Demanda con Elasticidad Unitaria $\left(\eta^{d}=-1\right)$, Demanda Inelástica $\left(\eta^{d}=-0,3\right)$. 
leña seca consumida sólo en el caso de demanda por leña seca elástica. Para este escenario se alcanzan valores de la proporción de leña seca consumida resultante de $22,2 \%, 24,4 \%$ y $26,5 \%$ para subsidios de $30 \%, 60 \%$ y $90 \%$ sobre la diferencia de precios, respectivamente (escenario I, II y III, Cuadro 3). Este ejercicio supone, naturalmente, que los consumidores encuentran en oferta suficiente la leña seca demandada al nuevo precio.

Calculamos el monto de recursos involucrados en los programas de subsidios considerados de acuerdo a la expresión presentada en la ecuación (8). Considerando que los mayores impactos sobre la proporción de leña seca consumida para los tipos de subsidio simulados se originaron con el escenario de demanda elástica, el gasto en subsidio calculado consideró sólo tales escenarios (ver Cuadro 4).

Igualmente evaluamos, por medio de la expresión en la ecuación (9) y el modelo de emisiones referido en la Sección 3, los posibles impactos de las medidas de incentivo al consumo de leña seca sobre la contaminación en Temuco y Padre Las Casas. Los resultados tanto para los niveles de emisiones calculados en cada escenario, como para el impacto diferenciado con relación al escenario base se presentan en el Cuadro 5. Con el propósito de tener un patrón de comparación se consideró también simular un escenario hipotético, y probablemente no factible, en que la proporción consumida de leña seca alcanza a 100\%. En este escenario

\section{CUADRO 4}

GASTO SEGÚN PROGRAMA DE SUBSIDIO AL CONSUMO DE LEÑA SECA Y ELASTICIDAD SELECCIONADA EN TEMUCO Y PADRE LAS CASAS

(Millones de pesos)

\begin{tabular}{lcccc}
\hline & \multicolumn{3}{c}{$\begin{array}{c}\text { Escenarios de subsidio } \\
\text { al diferencial de precios }\end{array}$} \\
\cline { 2 - 4 } & & I & II & III \\
\hline Gasto en subsidio a la demanda (mill. \$/año) & 49,6 & 108,9 & 178,0 \\
\hline
\end{tabular}

Fuente: Elaboración propia.

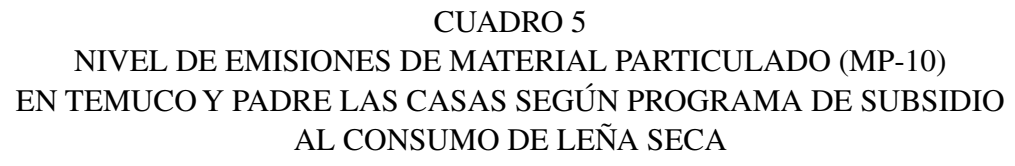

\begin{tabular}{|c|c|c|c|c|c|}
\hline \multirow[t]{2}{*}{ Emisiones } & \multirow[t]{2}{*}{ Caso base } & \multicolumn{3}{|c|}{$\begin{array}{l}\text { Escenarios de subsidio } \\
\text { al diferencial de precios }\end{array}$} & \multirow{2}{*}{$\begin{array}{l}\text { Caso ideal } \\
100 \% \text { leña } \\
\text { seca }\end{array}$} \\
\hline & & I & II & III & \\
\hline Emisiones (ton/año) & 3.351 & 3.329 & 3.307 & 3.287 & 2.566 \\
\hline$\Delta$ emisiones (ton/año) & & -22 & -44 & -64 & -785 \\
\hline
\end{tabular}

Fuente: Elaboración propia. 
la reducción máxima posible de emisiones de material particulado en Temuco y Padre Las Casas asciende a un total de 785 toneladas anuales, correspondiendo a un $23 \%$ de reducción sobre la situación base.

Los resultados obtenidos para el impacto sobre emisiones son más bien modestos, independiente de la profundidad del programa de subsidio considerado (note que se presentan resultados sólo para el caso de una demanda de leña seca elástica al precio). En el caso más ambicioso evaluado ( $90 \%$ de la brecha de precio entre leña seca y húmeda, escenario III, Cuadro 5) se alcanza sólo una modesta reducción de poco menos de $2 \%$ de emisiones anuales sobre el escenario base (64 toneladas menos $)^{23}$.

Finalmente, obtenemos indicadores de costo-efectividad calculados como cuocientes entre el gasto en subsidio y la reducción en las emisiones de material particulado logradas (ver Cuadro 6). Notamos que mientras más pequeño es el valor reportado, más costo efectivo es el instrumento, es decir, menor es el gasto en subsidio por tonelada reducida. Los resultados indican que el gasto por tonelada reducida se incrementa a medida que el programa de subsidio se torna más ambicioso como sugiere la ecuación (11). Por ejemplo, mientras el costo de reducir una tonelada al año con un programa de subsidio de $30 \%$ a la brecha de precio entre leña seca y húmeda (Escenario I) alcanza a 2,25 millones de pesos, el mismo costo asciende, con un programa de subsidio de $90 \%$ (Escenario I), a un monto de 2,77 millones de pesos. Este deterioro en el coeficiente costo-efectividad puede interpretarse de la siguiente manera: la reducción de emisiones lograda se genera sólo por el aumento diferencial en la proporción de leña seca utilizada, y no alcanza a compensar los gastos que involucran subsidios más agresivos que aplican al total de leña seca consumida.

\section{CUADRO 6 \\ COSTO EFECTIVIDAD SEGÚN PROGRAMA DE SUBSIDIO \\ AL CONSUMO DE LEÑA SECA Y ELASTICIDAD SELECCIONADA \\ EN TEMUCO Y PADRE LAS CASAS}

(Millones de pesos)

\begin{tabular}{lcccc}
\hline & \multicolumn{3}{c}{$\begin{array}{c}\text { Escenarios de subsidio } \\
\text { al diferencial de precios }\end{array}$} \\
\cline { 2 - 4 } & I & II & III \\
\hline Índice costo-efectividad (mill. \$/ton./año) & 2,25 & 2,50 & 2,77 \\
\hline
\end{tabular}

Fuente: Elaboración propia.

23 La dependencia lineal de la reducción de emisiones respecto al incremento en el consumo de leña seca postulada en la ecuación (10) se valida indirectamente con los resultados del Cuadro 5. Para ello debe tenerse en cuenta que debido a que el total de leña se mantiene constante, el incremento en la cantidad de leña seca es una función lineal de la variación del porcentaje de leña seca/húmeda consumida, presentado en el Cuadro 3. Como conclusión, la reducción de emisiones también dependerá linealmente de la variación del porcentaje mencionada. Esta linealidad puede observarse comparando directamente la primera fila del Cuadro 3 ("Elástica") con la segunda fila del Cuadro 5 (" $\Delta$ Emisiones"). 


\subsection{Subsidio a la oferta}

Considerando el precio de leña húmeda a nivel de productores (en porcentajes del $50 \%$ y $20 \%$ respecto del precio de la leña húmeda), la tasa de interés (estimada en un 1,4\%) y un tiempo de secado de 12 meses, se obtienen los siguientes precios de leña seca para los productores y los costos de oportunidad correspondientes (ver Cuadro 7).

\section{CUADRO 7 \\ ESCENARIOS DE COSTO DE OPORTUNIDAD DEL SECADO DE LA LEÑA PARA LOS PRODUCTORES \\ $\left(\$ / \mathrm{m}^{3}\right.$ estéreo)}

\begin{tabular}{cccc}
\hline & \multicolumn{3}{c}{$\begin{array}{c}\text { Escenarios de costo de oportunidad } \\
\text { para los productores del secado de la leña }\left(\$ / \mathrm{m}^{3} \text { estéreo) }\right.\end{array}$} \\
\cline { 2 - 4 } $\begin{array}{c}\text { del precio base de } \\
\text { leña húmeda } \\
\text { (sobre los } \$ 15.000)\end{array}$ & $\begin{array}{c}\text { Precio de leña } \\
\text { que obtienen los } \\
\text { productores }\end{array}$ & $\begin{array}{c}\text { Precio incrementado } \\
\text { considerando período } \\
\text { de secado }\end{array}$ & $\begin{array}{c}\text { Costo de oportunidad } \\
\text { asociado al periodo } \\
\text { de secado }\end{array}$ \\
\hline 50 & 7.500 & 8.900 & 1.400 \\
20 & 3.000 & 3.500 & 500 \\
\hline
\end{tabular}

Fuente: Elaboración propia en base a información provista por CONAMA-Región de la Araucanía. Nota: En el cálculo se utilizó una tasa de 1,4\% de interés mensual, a 12 meses.

Para estimar los costos totales de un programa de subvención al secado de la leña se utilizaron los mismos montos estimados de leña seca a consumir resultantes bajo los escenarios correspondientes al subsidio a la demanda. La simulación se realizó solamente para los estimados de consumo de leña seca resultantes del caso de una demanda elástica, pero considerando los tres niveles de subsidio a la brecha de precios. Enfatizamos que no se consideran los subsidios a la demanda y a la oferta en conjunto, sino que sólo se toman los montos estimados de consumo de leña seca del caso de demanda, para con ellos calcular la cantidad a subvencionar por el lado de la oferta. Esta coincidencia entre los montos de leña seca subvencionados, permite comparar más fácilmente ambos programas de subsidio (ver Sección 2.2). Para los escenarios consideramos (subsidio de 30\%, 60\% y 90\%), los volúmenes anuales de leña seca requeridos son de 110.152, 120.987 y 131.821 $\mathrm{m}^{3}$ estéreo, respectivamente (ver Cuadro 8).

De acuerdo a la ecuación (12), el costo total del subsidio al secado de leña resulta de multiplicar las cantidades anuales requeridas de leña seca por el costo de secado de un $\mathrm{m}^{3}$ estéreo para un periodo de 12 meses (Cuadro 7, Columna 4). Los resultados para los costos totales de secado de leña se presentan en el Cuadro 8.

Contrastando los resultados anteriores con los montos de reducción de emisiones presentados en el Cuadro 5, se calculan indicadores de costo efectividad para el subsidio del secado orientado a los productores (ver Cuadro 9). Los resultados del ejercicio muestran consistencia con la ecuación (13), ya que el indicador de costo efectividad va mejorando siempre que la agresividad del programa (monto 
de leña seca a subvencionar) aumente ( $s$ creciendo) o que el precio de la leña húmeda supuesto para los productores disminuya ( $\rho$ disminuyendo).

De acuerdo a lo señalado en la sección 2.2 la relación entre los índices de costo-efectividad de un programa de subsidio a la demanda (brecha de precios) o al secado, depende únicamente de la comparación entre el monto del subsidio $(s)$ y el costo de secado $(\rho)$. Por ejemplo, en el caso de un subsidio a la brecha de precios de $30 \%$ ( $s=\$ 450 \mathrm{~m}^{3}$ estéreo) y de un costo de secado $\rho$ de $\$ 1.400 \mathrm{~m}^{3}$ estéreo, mientras el coeficiente de costo-efectividad para el subsidio a la demanda asciende a 2,5 millones de pesos por tonelada reducida, el mismo indicador para el programa de subsidio al secado alcanza a 7 millones de pesos por tonelada reducida. En tanto que, en el caso de un subsidio a la demanda de $90 \%\left(s=\$ 1.350 \mathrm{~m}^{3}\right.$ estéreo) y un costo de secado $\rho$ de $\$ 500 \mathrm{~m}^{3}$ estéreo, se invierte el orden de magnitud de los indicadores costo-efectividad, alcanzado éstos a 2,9 y 1,0 millones por tonelada reducida, para el subsidio a la demanda y al secado, respectivamente.

\section{CUADRO 8 \\ COSTOS TOTALES DE SUBSIDIAR TODA LA LEÑA SECA A CONSUMIR PARA DISTINTOS ESCENARIOS DE CONSUMO \\ Y DE PRECIOS AL PRODUCTOR \\ (Millones de pesos al año, tiempo secado 12 meses)}

\begin{tabular}{crrr}
\hline & \multicolumn{3}{c}{ Escenarios de subsidio al secado } \\
\cline { 2 - 4 } & \multicolumn{1}{c}{ I } & II & III \\
\hline Volumen de leña seca a subsidiar $\left(\mathrm{m}^{3}\right.$ estéreo) & 110.152 & 120.987 & 131.821 \\
\hline Precio leña húmeda & Costos totales del subsidio al secado \\
zona rural & \multicolumn{3}{c}{ (mill. \$ al año) } \\
\hline$\$ 7.500$ & 154 & 169 & 185 \\
$\$ 3.000$ & 55 & 60 & 66 \\
\hline
\end{tabular}

Fuente: Elaboración propia.

Nota: Todos los escenarios suponen una demanda elástica $\left(\eta^{d}=-4\right)$.

\section{CUADRO 9 \\ INDICADORES COSTO-EFECTIVIDAD PARA PROGRAMA DE SUBSIDIO A LA DEMANDA Y OFERTA DE LEÑA (ETAPA DE PRODUCCIÓN Y SECADO)}

\begin{tabular}{cccc}
\hline & \multicolumn{3}{c}{ Escenarios de subsidio al secado } \\
\cline { 2 - 4 } Precio leña húmeda & I & II & III \\
\cline { 2 - 4 } zona rural & & \multicolumn{3}{c}{$\begin{array}{c}\text { Índice costo-efectividad del subsidio al secado } \\
\text { (millones de pesos) }\end{array}$} \\
\hline$\$ 7.500$ & 7,0 & 3,9 & 2,9 \\
$\$ 3.000$ & 2,5 & 1,4 & 1,0 \\
\hline
\end{tabular}

Fuente: Elaboración propia. 


\subsection{Fiscalización}

Con la intención de simplificar los cálculos se fijó el monto de la sanción $\phi$ por cada $\mathrm{m}^{3}$ estéreo de leña húmeda transportada, como la diferencia de precios entre la leña seca y húmeda en la zona rural. En concordancia con nuestro análisis anterior para el caso del subsidio al secado esta diferencia es exactamente igual al costo de oportunidad del secado $(\rho=\phi)$. Según la expresión de la ecuación (19) esta elección de la sanción vuelve al indicador de costo efectividad de la fiscalización independiente del costo de secado, y por ende también de la diferencia de precios de leña en la zona rural.

Necesitamos fijar también, de acuerdo al modelo de la sección 2.3, los niveles de precios de la leña seca y húmeda en las zonas rural y urbana. Los precios de la leña en la zona urbana han sido calculados para todo el estudio como de $\$ 15.000$ para la leña húmeda y $\$ 16.500$ para la seca. Respecto a los precios en la zona rural, y teniendo en cuenta lo explicado en el párrafo anterior, consideraremos sólo uno de los escenarios utilizados en el análisis del subsidio a la oferta, el que corresponde a niveles de precio para productores de $\$ 7.500$ y $\$ 8.900$ para la leña húmeda y seca, respectivamente (ver Cuadro 7). Esta elección significa un costo de secado (y de sanción) de $\$ 1.400 \mathrm{~m}^{3}$ estéreo.

Los escenarios para las simulaciones sobre fiscalización también requieren prefijar los niveles deseados de cumplimiento $\delta$ de la regulación. Como hemos mencionado previamente, la situación base se ha fijado para $\bar{\delta}=0.8$. Para poder hacer comparables los resultados de costo efectividad de las distintas simulaciones se utilizaron los montos estimados de leña seca a consumir obtenidos bajo los mismos escenarios que para los cálculos de subsidio a la demanda y a la oferta. La simulación en el caso de la fiscalización se limitó entonces sólo a los escenarios caracterizados por una demanda elástica y tres alternativas de subsidio al diferencial de precios considerados. Estos escenarios resultan en distintas proporciones de leña seca-leña húmeda a utilizar. Para subsidios sobre el diferencial de precios entre leña seca y húmeda de 30\%, 60\% y 90\% (escenarios I, II y III), la porción de leña húmeda a transportar es de 77,8\%,75,6\% y 73,5\%, respectivamente (ver Cuadro 10, primera fila).

Utilizando los ajustes del modelo mencionados en el Apéndice y la expresión en la ecuación(16) podemos calcular en cada escenario considerado un nivel de fiscalización expresado en la probabilidad $(\pi)$ de auditar o controlar la entrada de leña. Las probabilidades requeridas para inducir los niveles de cumplimiento mencionados anteriormente son presentadas en la segunda fila del Cuadro $10^{24}$.

El costo total de fiscalización se calcula, de acuerdo a la ecuación (18), multiplicando el costo de un equipo de fiscalización (\$5.600.000) por el número

\footnotetext{
24 Hacemos notar que no se dispone de información sobre actividades de fiscalización orientadas al cumplimiento de estándares de calidad de la leña. En tal sentido, el nivel de fiscalización $\pi$, debe ser interpretado como una sugerencia respecto al esfuerzo efectivo de control que se requiere desplegar y no puede ser corroborado con datos de la práctica. Concluimos por tanto que, con la información actualmente disponible, sería inapropiado intentar sacar conclusiones válidas respecto a la factibilidad de implementar los niveles de esfuerzo de fiscalización obtenidos.
} 
esperado de equipos de fiscalización requeridos para inducir cumplimiento de la norma considerada. Los resultados en relación a los costos totales se presentan en la tercera fila del Cuadro 10. Como es de esperar mientras se pretenda hacer cumplir a un nivel más alto la regulación sobre la prohibición de la comercialización, i. e. reducir la proporción de leña que se transporta húmeda, es necesario imponer una probabilidad de auditoría mayor sobre el sistema.

\author{
CUADRO 10 \\ ESFUERZO, COSTOS TOTALES \\ E ÍNDICE COSTO-EFECTIVIDAD DE FISCALIZACIÓN
}

\begin{tabular}{lccc}
\hline & \multicolumn{3}{c}{$\begin{array}{c}\text { Escenarios considerados } \\
\text { para la fiscalización }\end{array}$} \\
\cline { 2 - 4 } & I & II & III \\
\hline Porcentaje de leña que se transporta húmeda $(\delta)$ & 77,8 & 75,6 & 73,5 \\
Nivel de fiscalización requerido $(\pi(\delta))$ & 0,0275 & 0,0550 & 0,0813 \\
Costos totales de la fiscalización (mill. \$ al año) & 3,08 & 6,16 & 9,10 \\
Índice costo-efectividad (mill. \$) de la fiscalización & 0,14 & 0,14 & 0,14 \\
\hline
\end{tabular}

Fuente: Elaboración propia.

Nota: Todos los escenarios suponen una demanda elástica $\left(\eta^{d}=-4\right)$. Precio de leña húmeda asumido en zona rural $\$ 7.500$.

Comparando los costos de fiscalización con los montos de reducción de emisiones presentados en el Cuadro 5 de la sección anterior, podemos calcular indicadores de costo efectividad para un sistema de fiscalización que induzca el cumplimiento parcial de la norma sobre prohibición de la comercialización de leña húmeda. Los resultados se presentan en el Cuadro 10, cuarta fila, y muestran que los indicadores de costo efectividad obtenidos son superiores al ser comparados con los otros instrumentos. Específicamente, en el caso de fiscalización, el indicador de costo-efectividad alcanza a 0,14 millones de pesos por tonelada reducida, resultado que se diferencia de manera importante de los indicadores obtenidos para los casos de subsidios; los cuales resultan en coeficientes costo-efectividad entre uno y siete millones de pesos por tonelada reducida (ver Cuadro 9 y Cuadro 10). Los resultados corroboran el indicador de costo-efectividad derivado en la sección 2.3 y presentado en la ecuación (19), en el sentido que el coeficiente de costo-efectividad para el programa de fiscalización no depende del nivel de cumplimiento $(\delta)$ deseado.

Los resultados comparativos en cuanto a costo-efectividad fueron presentados para varios escenarios de simulación y en todos ellos la fiscalización genera indicadores de costo-efectividad mejores que aquellos originados por los subsidios evaluados. Adicionalmente, se realizaron estudios de sensibilidad para diversos parámetros tales como el tiempo de secado, monto de subsidio, etc. El resultado de este análisis de sensibilidad indica que el mensaje central que emerge en cuanto 
a la comparación de costo-efectividad es invariable. Con vistas a mantener una razonable extensión de nuestro trabajo y para no afectar la lectura del mismo, hemos desistido de la presentación de todos los resultados de simulación realizados.

\section{CONCLUSIONES}

El análisis respecto al uso de incentivos económicos para apoyar la utilización de leña seca, sugiere que los esquemas de subsidio a la oferta son más costo-efectivos que aquellos que consideran subsidios a la demanda de leña. Sin embargo, el valor de la leña húmeda que observan los productores influye negativamente sobre los resultados de costo-efectividad del subsidio al secado de leña. Este efecto, puede llevar a revertir la ventaja de este subsidio para escenarios en los cuales éste sea sobre el diferencial de precios en el consumo.

Basados en supuestos sobre la elasticidad de la demanda de leña seca se ha calculado la costo-efectividad de programas de subsidio orientados tanto a la demanda como a la oferta de leña seca. Los resultados indican que el gasto en subsidio a la demanda por tonelada anual reducida crece de manera acelerada cuando aumenta la profundidad del programa de subsidio. Se observa inclusive un deterioro progresivo en el coeficiente costo-efectividad, que se atribuye a que los mayores gastos necesarios para subvencionar la demanda no se logran compensar adecuadamente con la reducción de emisiones lograda por el aumento diferencial (debido al programa de subsidio) en la proporción de leña seca utilizada.

Por el lado de la oferta sólo se hicieron estimaciones primarias de los costos totales de un programa de subsidio del secado orientado a los productores. En este caso el indicador de costo-efectividad muestra una dependencia directa respecto al monto total de leña a subvencionar e inversa respecto al precio realizado para la leña húmeda por parte de los productores.

Por último se observa que articular esfuerzos de fiscalización es mucho más costo-efectivo que cualquier otro de los mecanismos económicos estudiados. Esto se debe a la acción casi directa y a bajo costo de la fiscalización, sobre el parámetro que ciertamente decide sobre los niveles de emisión: la proporción final de leña húmeda utilizada.

\section{REFERENCIAS}

Barna, M. y N. Gimson (2002), "Dispersion Modeling of Wintertime Particulate Pollution Episode in Christchurch, New Zealand", Atmospheric Environment 36(21): 3531-3544.

Barnes, D., K. Krutilla y W. Hyde (2005), "Household Fuel Choice and Consumption", Capítulo 3 en The Urban Household Energy Transition. Social and Environmental Impacts in the Developing World, Resources for the Future (RFF) Press Book. 
Becker, G. (1968). “Crime and Punishment: An Economic Approach”, Journal of Political Economy 76: 169-217.

Celis, J., R. Flocchini, O. Carvacho, J. Morales, C. Zaror, J. Insunza y M. Pineda (2006), "Analysis of Aerosol Particles and Coarse Particulate Matter Concentrations in Chillan, Chile, 2001-2003", Journal of the Air and Waste Management 56: $152-158$.

Celis, J., J. Morales, C. Zaror y J. Insunza (2004), "Study of the Particulate Matter $\mathrm{PM}_{10}$ in the Atmosphere of Chillan, Chile", Chemosphere 54: 541-550.

CONAMA-DICTUC (2007), "Actualización del Inventario de Emisiones de Contaminantes Atmosféricos en la Región Metropolitana 2005", mimeo.

CONAMA-DICTUC (2008), "Actualización del Inventario de Emisiones Atmosféricas en las Comunas Temuco y Padre Las Casas", mimeo.

CONAMA (2007a), "Anteproyecto de Plan de Descontaminación Atmosférico para las Comunas de Temuco y Padre Las Casas". Resolución Exenta No 1190, mimeo.

CONAMA (2007b), "Reporte Contenido de Humedad de La Leña en Temuco y Padre Las Casas”. Informes 2004-2007. CONAMA IX Región de La Araucanía, mimeo.

Cooke, P., G. Köhlin y W. Hyde (2008), "Fuelwood, Forests and Community ManagementEvidence from Household Studies", Environment and Development Economics 13: 103-135.

Gómez-Lobo, A. (2005), "El Consumo de Leña en el Sur de Chile:¿ Por Qué Nos Debe Preocupar y Qué se Puede Hacer?, Revista Ambiente y Desarrollo, N²1(3):43-47.

Heltberg, R. (2005), "Factors Determining Household Fuel Choice in Guatemala". Environment and Development Economics 10(3):337-361.

Heltberg, R., T. Channing y N. Udaya (2000), "Fuelwood Consumption and Forest Degradation: A Household Model for Domestic Energy Substitution in Rural India", Land Economics 2: 213-232.

Katzner, D. (1988), "Markets in Isolation", Capítulo 6, en Walrasian Microeconomics. An Introduction to the Economic Theory of Market Behavior. Addison-Wesley Publishing Company.

Krumdieck, S. (2008), "Waste Wood to Heat: Beyond Technology to Quality of Life in Christchurch", The Institution of Professional Engineers of New Zealand, mimeo.

Navarrro C., J. Pinares y J. Castillo (2005), "Estudio de Secado de Leña y Equivalencias de Unidades de Comercialización". Informe Final para CONAMA IX REGIÓN, mimeo.

Nicholson, W. (1997), Teoría Microeconómica: Principios Básicos y Aplicaciones. McGrawHill, $1^{\text {a }}$ Edición.

Otero, L., M. Lobos, A. Vera y T. Kausel (2004). Estudio: “Generación de Antecedentes para la Implementación de un Sistema Nacional de Certificación de Leña. Propuesta de un Sistema Nacional de Certificación de Leña”. Informe Final, CONAMA IX Región de La Araucanía.

Sanhueza, P., M. Fernández, P. Pastene y M. Torreblanca (2006), "Análisis de Medidas para Incorporar al Plan de Descontaminación Atmosférica de Temuco y Padre Las Casas". Informe Final Proyecto BIP 30039981-0. Estudio desarrollado para Gobierno Regional de La Araucanía - CONAMA Región de La Araucanía.

Universidad de Concepción (2002), Estudio: "Priorización de Medidas de Reducción de Emisiones por Uso Residencial de Leña para la Gestión de la Calidad del Aire en Temuco y Padre Las Casas". Informe Final contrato 14-22-008/01 para CONAMA IX Región de La Araucanía. 


\section{APÉNDICE}

En el texto central se introduce la función $d(\delta)$ que representa el porcentaje de leña húmeda que puede comercializarse como seca. Por su naturaleza, esta cantidad debe cumplir el siguiente requisito.

\section{(A.1) $d(\delta) \in[0,1] \forall \delta \in[0,1]$}

Suponemos en adelante que $d(\delta)=a+b \delta$. Esta hipótesis hace a la función de beneficio $B(\delta)$ cuadrática y cóncava si $\left(P_{U S}-P_{U H}\right) b<025$.

De acuerdo a la discusión en el texto central, la función $B(\delta)$ de beneficio debe alcanzar su máximo en la situación base $\delta=\bar{\delta}$, con $0<\bar{\delta}<1$. Esto requiere la concavidad de $B(\delta)$ y como $\left(P_{U S}-P_{U H}\right)>0$ necesitamos entonces que $b<0$. Bajo este supuesto (A.1) se reduce a las tres siguientes condiciones:

$$
\text { (A.2) } b<0 \quad a \geq-b \quad a \leq 1
$$

Usando la ecuación (12) en el texto central en conjunto con el supuesto respecto a la linealidad de la función $d$, para el escenario base obtenemos la relación:

(A.3) $\bar{\delta}=\frac{\left(\left(P_{U S}-P_{U H}\right)-\left(P_{R S}-P_{R H}\right)\right)-(a+b \bar{\delta})\left(P_{U S}-P_{U H}\right)}{\left(P_{U S}-P_{U H}\right) b}$

Esta condición es equivalente a que bajo ausencia de fiscalización el máximo beneficio se obtenga en el valor $0<\bar{\delta}<1$ supuesto como escenario base. La condición (A.3) puede re-escribirse como:

$$
a=\frac{\left(P_{U S}-P_{U H}\right)(1-2 b \bar{\delta})-\left(P_{R S}-P_{R H}\right)}{\left(P_{U S}-P_{U H}\right)}
$$

El resultado principal de este apéndice son condiciones sobre los datos del modelo que garantizan un ajuste razonable de $d(\delta)=a+b \delta$.

\footnotetext{
25 En nuestro modelo, y según se establece en la sección $2.3, d(\delta)$ representa el porcentaje de leña húmeda que se podría comercializar como seca. Nótese que el valor de $d(\delta)$ no tiene relevancia práctica en el caso $\delta=0$, pues no interviene en el cálculo del beneficio asociado (ver ecuación (14)). La función $d(\delta)$ refleja cuán probable es poder aprovecharse de la ventaja de información subyacente en dependencia de la cantidad total de leña húmeda transportada. Si $d(0)=0$, la hipótesis de que $d^{\prime}(\delta) \leq 0$ entregaría un valor negativo para $d(\delta)$, lo cual haría imposible interpretar éste como un porcentaje. Por otra parte, la hipótesis de que $d^{\prime}(\delta) \leq 0$, en el caso de $d(\delta)$ lineal, asegura que las soluciones al problema planteado en la ecuación (15) no sean siempre extremas $(\delta=0$ ó $\delta=1)$, lo cual rescata el sentido práctico de la modelación.
} 


\section{Proposición:}

Sean $0<\bar{\delta}<1,\left(P_{U S}-P_{U H}\right)>0$ y $\left(P_{R S}-P_{R H}\right)>0$ parámetros fijos. Si se satisface la condición

(A.5) $2 \bar{\delta} \geq \frac{\left(P_{R S}-P_{R H}\right)}{\left(P_{U S}-P_{U H}\right)}$

entonces pueden encontrarse parámetros $a$ y $b$ que satisfagan las condiciones (A.2) y (A.4).

\section{Demostración:}

La igualdad (A.4) pone $a$ en función de $b$, así que solo debemos sustituirla en las desigualdades de (A.1) y ver si se mantienen consistentes. Supongamos en lo adelante entonces que $a$ tiene la expresión de (A.4). En ese caso la condición $a \geq-b$ se escribe como:

(A.6) $\frac{\left(P_{U S}-P_{U H}\right)-\left(P_{R S}-P_{R H}\right)}{\left(P_{U S}-P_{U H}\right)} \geq b(2 \bar{\delta}-1)$

Por otra parte, también utilizando (A.4) se escribe $a \leq 1$ como

(A.7) $-\frac{\left(P_{R S}-P_{R H}\right)}{2 \bar{\delta}\left(P_{U S}-P_{U H}\right)} \leq b$

Notemos ahora que si tomamos como valor de $b$, la parte izquierda de (A.7), o sea,

(A.8) $b=-\frac{\left(P_{R S}-P_{R H}\right)}{2 \bar{\delta}\left(P_{U S}-P_{U H}\right)}$

entonces se cumplen automáticamente las condiciones $b<0$ y $a \leq 1$. Falta por comprobar que (A.6) es consecuencia de (A.8) y (A.5). Pero sustituyendo (A.8) en (A.6) se obtiene:

(A.9) $\frac{\left(P_{U S}-P_{U H}\right)-\left(P_{R S}-P_{R H}\right)}{\left(P_{U S}-P_{U H}\right)} \geq-\frac{(2 \bar{\delta}-1)\left(P_{R S}-P_{R H}\right)}{2 \bar{\delta}\left(P_{U S}-P_{U H}\right)}$

que mediante transformaciones elementales se convierte exactamente en (A.6). 